\title{
ВНЕДРЕНИЕ В КЛИНИЧЕСКУЮ ПРАКТИКУ НОВЫХ ИННОВАЦИОННЫХ МИКРОХИРУРГИЧЕСКИХ МЕТОДОВ ОПЛОДОТВОРЕНИЯ ООЦИТОВ IN VITRO
}

\author{
Саватеева Н.А., Мун Т.В., Витязева И.И.
}

ФГБУ «НМИЦ эндокринологии» Минздрава России

\begin{abstract}
Изменения в структуре сперматозоидов являются важным фактором, приводящим к нарушению фертильности мужчин. До 7\% мужчин в общей популяции испытывают проблемы с зачатием вследствие органических нарушений сперматозоидов, в последующем влияющих на их функциональное состояние. Использование современных молекулярно-биологических, биохимических, цитогенетических методов исследований способно расширить понимание о том, какие именно структурные повреждения вносят основной вклад в развитие бесплодия у мужчин, а также может являться не только первым шагом для разработки таргетной терапии, но и усовершенствования методов оплодотворения, улучшая качество выбора сперматозоидов.

Методы вспомогательных репродуктивных технологий (ВРТ) широко применяются при лечении мужского бесплодия. С внедрением микрохирургической техники интрацитоплазматической инъекции сперматозоида в цитоплазму яйцеклетки (ИКСИ), стало возможным проводить лечение бесплодия при тяжелых степенях патозооспермии, вплоть до оплодотворения единичными эпидимальными и тестикулярными сперматозоидами. При стандартном методе ИКСИ, применяемым с 1995 г., селекция сперматозоидов осуществляется на основе визуальной оценки их морфологии и подвижности при 400-кратном увеличении. Однако такой подход не всегда позволяет оценить отсутствие повреждения внутренних структур сперматозоида и его потенциальный вклад в доимплантационное развитие эмбриона.

В настоящее время разработано два основных метода селекции сперматозоидов для проведения оплодотворения: метод ПИКСИ приближает выбор сперматозоида к физиологическому (естественному) отбору - помимо подвижности сперматозоида и его внешнего неизмененного строения, возможно использовать свойство сперматозоида прикрепляться к блестящей оболочке ооцита, где локализованы рецепторы к гиалуронану, что свидетельствует о зрелости хроматина в его головке, посредством проведения теста с гиалуронаном (НВА-тест); метод ИМСИ основан на селекции сперматозоидов по морфологическим критериям при большом оптическом и цифровом увеличении до $\times 7500$. В ходе ИМСИ выбираются только самые качественные сперматозоиды для непосредственного инъецирования в яйцеклетку.

Однако каждый из этих методов обладает рядом недостатков: при использовании метода ПИКСИ в качестве метода селекции при оплодотворении может быть отобран сперматозоид зрелый по качеству, но с измененной морфологией в силу того, что используется небольшое увеличение; при методе ИМСИ можно отобрать идеальный сперматозоид по морфологии, но он окажется незрелым.

ПИМСИ - новый инновационный двухэтапный метод оплодотворения, базирующийся на методе ИКСИ, основывается на качественной селекции сперматозоидов по морфологическим признакам с использованием раствора гиалуронана (ПИКСИ), а ИМСИ позволяет производить отбор сперматозоидов при оптическом и цифровом увеличении до х7500. Данный метод отбора сперматозоидов позволит существенно повысить эффективность программ ЭКО в случаях тяжелого мужского фактора бесплодного брака.
\end{abstract}

